\title{
Marcadores sorológicos das hepatites B e C em doadores de sangue do Hemocentro de Ribeirão Preto, SP
}

\author{
Hepatitis B and C serologic markers in blood \\ donors of the Ribeirão Preto Blood Center
}

\author{
Vanderléia Bárbaro Valente ${ }^{1}$, Dimas Tadeu Covas ${ }^{2}$ \\ e Afonso Dinis Costa Passos ${ }^{3}$
}

\begin{abstract}
RESUMO
Este estudo envolveu 25.891 doadores de sangue que compareceram pela primeira vez ao Hemocentro de Ribeirão Preto, entre 23/06/1996 e 22/06/2001. Seu objetivo foi estudar a positividade de marcadores sorológicos das hepatites B e $C$ em testes de triagem e estimar a prevalência de infecção atual ou pregressa pelos vírus de ambas as hepatites, através da análise dos resultados de testes confirmatórios. Estudaram-se dados do Hemocentro e do Hospital das Clínicas da Faculdade de Medicina, coletando-se informações referentes ao doador e aos resultados dos testes sorológicos. A população foi composta majoritariamente por homens (83,6\%) de 26 a 45 (64\%) anos de idade. Os valores de positividade nos testes da triagem foram 0,6\% (IC $\left.{ }_{95 \%}: 0,54-0,72\right)$ para o HBsAg e 1,2\% (IC $\left.C_{95 \%}: 1,02-1,28\right)$, para o anti-HCV. Os valores da prevalência, nos testes confirmatórios, foram $0,2 \%$ (IC $\left.C_{95 \%}: 0,16-0,28\right)$, para a hepatite $B$, e $0,3 \%\left(I C_{95 \%}: 0,24-0,38\right)$ para a hepatite $C$.
\end{abstract}

Palavras-chaves: Doadores de sangue. Hepatites. Hepatite B. Hepatite C. Marcadores sorológicos. Prevalência.

\begin{abstract}
The investigation involved 25,891 blood donors who attended for the first time the Blood Center of Ribeirão Preto, Brazil, between Jun 23, 1996 and Jun 22, 2001. The objective was to study the proportion of positive serological markers for hepatitis $B$ and $C$ at the initial screening tests and to estimate the prevalence of such infections through the analysis of confirmatory tests, carried out at the University Hospital as a part of the patients evaluation. Data from the donors and laboratory results were obtained from the records of both the Blood Bank and the Hospital. The population of donors was mainly composed by males (83.6\%) and by individuals between 26 and $45(64 \%)$ years of age. The proportions of positive results in screening tests were $0.6 \%\left(\mathrm{CI}_{95 \%}: 0.54-0.72\right)$ for HBsAg and $1.2 \%\left(\mathrm{CI}_{95 \%}: 1.02-1.28\right)$ for anti-HCV. Confirmatory testes showed values of prevalence of $0.2 \%\left(C I_{95 \%}: 0.16-0.28\right)$ for hepatitis $B$ and $0.3 \%\left(I C_{95 \%}: 0.24-0.38\right)$ for hepatitis $C$.
\end{abstract}

Key-words: Blood donors. Hepatitis. Hepatitis B. Hepatitis C. Serologic markers. Prevalence.

As infecções pelos vírus da hepatite B (VHB) e da hepatite C (VHC) constituem grave problema de saúde pública em diferentes partes do mundo ${ }^{2} 1428$, porque se associam a elevado grau de cronificação e podem evoluir para cirrose hepática e carcinoma hepatocelular ${ }^{2425}$. No Brasil, calculase que existam, no mínimo, 3 milhões de portadores crônicos de cada um desses vírus, com estudos em doadores de sangue mostrando valores de prevalência de infecção crônica por cada um deles variando de $1 \%$ a $10 \%$, conforme a regiã $0^{15}$.

O VHB é transmitido por transfusão de sangue e hemoderivados, transplante de órgãos, hemodiálise, aleitamento materno, contaminação de agulhas, seringas e materiais intravenosos, e por via sexual, destacando-se 0 papel de fluidos orgânicos, como o sêmen e a saliva ${ }^{2356}$.

\footnotetext{
1. Hemocentro de Ribeirão Preto, Ribeirão Preto, SP. 2. Departamento de Clínica Médica da Faculdade de Medicina de Ribeirão Preto da Universidade de São Paulo, Ribeirão Preto, SP. 3. Departamento de Medicina Social da Faculdade de Medicina de Ribeirão Preto da Universidade de São Paulo, Ribeirão Preto, SP. Endereço para correspondência: Dra. Vanderléia Bárbaro Valente. Travessa Mossoró 26, Ipiranga, 14055-538 Ribeirão Preto, SP, Brasil.

Tel: $55163630-4841$

e-mail: labsor@pegasus.fmrp.usp.br

Recebido para publicação em 31/8/2004

Aceito em 20/8/2005
} 
0 vírus da hepatite $C$ é transmitido por transfusão de sangue e hemoderivados, hemodiálise, contaminação de agulhas, seringas e materiais intravenosos, e, secundariamente, por via sexual ${ }^{3}$. A hepatite $\mathrm{C}$ é a principal forma de hepatite transmitida por transfusão de sangue e hemoderivados, sendo o VHC encontrado principalmente no sangue total, papa de hemácias, plaquetas, plasma e, especialmente, nos concentrados de fatores da coagulação $0^{22}$.

A lei 7.649, de 25 de janeiro de 1988, estabeleceu a obrigatoriedade do cadastramento de doadores de sangue e a realização de exames laboratoriais para hepatite $\mathrm{B}$, sífilis, doença de Chagas, malária e aids ${ }^{8}$. Em 1993, a Portaria $1.376^{17}$ normalizou as práticas hemoterápicas no Brasil, tornando obrigatória também a inclusão de testes para anticorpos contra o VHC nos exames de triagem. Essas medidas reduziram a transmissão dessas duas formas de hepatite por transfusão de sangue e hemoderivados ${ }^{10}$.

Os reagentes atualmente empregados na triagem sorológica das hepatites $\mathrm{B}$ e C em bancos de sangue exibem boa sensibilidade e especificidade. Todavia, ao serem aplicados em populações onde são baixos os valores de prevalência, geram um percentual considerável de resultados falso-positivos. Faculta-se aos serviços de hemoterapia a realização de testes confirmatórios ou complementares, e, nos casos em que eles não são realizados, os doadores positivos deverão ser encaminhados a serviços especializados de acompanhamento ${ }^{17} 19$.

Desde setembro de 1992, os doadores do Hemocentro do Hospital das Clínicas da Faculdade de Medicina de Ribeirão Preto da Universidade de São Paulo (HCFMRP-USP), positivos para as hepatites B e C, são encaminhados ao Núcleo de Vigilância Epidemiológica (NVE) desse hospital. Depois de entrevistados por uma assistente social - quando se levantam informações de natureza demográfica e social e se investigam os fatores de risco de adquirir hepatites virais -, os doadores são encaminhados ao Ambulatório de Hepatites (AH) onde, num primeiro momento, passam por uma investigação que visa à confirmação diagnóstica e ao acompanhamento especializado, quando necessário ${ }^{1820}$.

O conhecimento da distribuição das hepatites B e C na clientela do Hemocentro de Ribeirão Preto é limitado em função de inexistência de estudos prévios sobre o tema. Assim, os objetivos deste trabalho foram estudar a positividade de marcadores sorológicos dessas hepatites em testes de triagem e estimar as prevalências de infecção atual ou pregressa, através da análise dos resultados de testes confirmatórios, na população de doadores que comparecem pela primeira vez ao Serviço.

\section{MATERIAL E MÉTODOS}

Foram estudados todos os indivíduos que doaram sangue no Hemocentro de Ribeirão Preto entre 23/6/1996 e 22/6/2001, levantando-se informações disponíveis no próprio Serviço e no HCFMRP-USP (NVE, AH, Serviço de Arquivo Médico e Laboratório de Gastroenterologia). As informações coletadas referiam-se a características demográficas dos doadores e resultados de exames sorológicos (HBsAg, anti-HBc total, anti-HBs, HBV-DNA, anti-HCV, Imunoblot para HCV e RNA-HCV).

Para a detecção dos marcadores para a hepatite B e C, nos testes de triagem, os procedimentos técnicos referentes aos ensaios foram realizados conforme especificações dos fabricantes dos reagentes e dos equipamentos, utilizando-se os kits comerciais rotineiramente adotados pela Hemorrede e pelo HCFMRP-USP, a seguir mencionados:

Determinação do HBsAg: Elisa Hepanostika ${ }^{\circledR}$, HBsAg UniForm II (Organon Teknika) e Auzyme Monoclonal EIA® (Abbott). Determinação do anti-HBc total: Elisa Hepanostika ${ }^{\circledR}$ anti-HBc Uni-Form II (Organon Teknika) e Corzyme EIA® (Abbott). Determinação do anti-HBs: Ausab EIA® (Abbott). Determinação do anti-HCV: HCV 3.0 IMX ® (Abbott); EIA 3.0 HCV ${ }^{\circledR}$ (Abbott) e Ortho HCV 3.0® Test Systems with Enchaved Save (Ortho).

A confirmação dos resultados de triagem para o VHB foi realizada através da repetição da teste para o HBsAg e da busca do HBV-DNA (Amplicor HBV monitor ${ }^{\text {TM }}$ test ${ }^{\circledR}$ - Roche). A confirmação dos resultados de triagem para o VHC foi realizada através da repetição do anti-HCV e da realização do RibaImunoblot (Chiron Riba HCV 3.0 SIA) e/ou da determinação do RNA-HCV (Amplicor Hepatitis C vírus Test, version 2.0®Roche). Em todas as situações foram seguidos os procedimentos técnicos recomendados pelos fabricantes.

Os dados obtidos foram codificados e digitados num banco de dados, utilizando-se o programa Epi-Info 6.04b7 . Posteriormente, foram transportados para um pacote estatístico (Stata 6.0) ${ }^{27}$ e submetidos à análise. Os resultados sorológicos foram cotejados com as variáveis individuais estudadas, buscando-se possíveis associações, que foram testadas mediante o emprego do teste qui-quadrado.

Este projeto foi aprovado pelo Comitê de Ética em Pesquisa Médica do HCFMRP-USP.

\section{RESULTADOS}

A distribuição dos primodoadores segundo a faixa etária e o sexo (Tabela 1) mostra amplo predomínio dos homens sobre as mulheres, com percentuais respectivamente iguais a $83,6 \%(21.639 / 25.891)$ e $16,4 \%(4.252 / 25.891)$. Com referência à idade, predominaram as faixas de 26 a 35 e de 36 a 45 anos, para ambos os sexos. No conjunto, observaramse percentuais consideravelmente menores nas faixas etárias de 18 a $25(17,8 \%)$ e de 46 a 60 (17\%) anos.

Conforme mostra a Tabela 2, o anti-HBc foi o marcador mais freqüente nos testes de triagem, estando presente em $2.250(8,7 \%)$ indivíduos. A comparação entre o HBsAg e 0 anti-HCV revela que o segundo apresentou positividade aproximadamente duas vezes maior que o primeiro $(1,2 \%$ e $0,6 \%$, com intervalos de confiança (95\%) respectivamente iguais a 1,02-1,28 e 0,54-0,72). Apenas 2 indivíduos mostraram-se simultaneamente positivos para estes dois últimos marcadores. 
Tabela 1 - Distribuição dos primodoadores segundo faixa etária e sexo. Hemocentro de Ribeirão Preto, 1996/2001.

\begin{tabular}{|c|c|c|c|c|c|c|}
\hline \multirow[b]{3}{*}{ Faixa etária (anos) } & \multicolumn{6}{|c|}{ Sexo } \\
\hline & \multicolumn{2}{|c|}{ feminino } & \multicolumn{2}{|c|}{ masculino } & \multicolumn{2}{|c|}{ total } \\
\hline & $\mathrm{n}^{0}$ & $\%$ & $\mathrm{n}^{0}$ & $\%$ & $\mathrm{n}^{0}$ & $\%$ \\
\hline $18-25$ & 1.002 & 23,6 & 3.607 & 16,7 & 4.609 & 17,8 \\
\hline $26-35$ & 1.483 & 34,9 & 7.714 & 35,6 & 9.197 & 35,5 \\
\hline $36-45$ & 1.081 & 25,4 & 6.307 & 29,1 & 7.388 & 28,5 \\
\hline $46-60$ & 632 & 14,9 & 3.774 & 17,4 & 4.406 & 17,0 \\
\hline Outras* & 54 & 1,3 & 237 & 1,1 & 291 & 1,0 \\
\hline Total & 4.252 & 100,0 & 21.639 & 100,0 & 25.891 & 100,0 \\
\hline
\end{tabular}

* Inclui indivíduos que por autorização médica, em virtude de situação especial, doaram sangue com idade inferior a 18 anos e superior a 60 anos

Tabela 2 - Distribuição dos primodoadores segundo resultados dos testes para as hepatites B e C realizados na triagem. Hemocentro de Ribeirão Preto, 1996/2001.

\begin{tabular}{|c|c|c|c|c|c|c|}
\hline & \multicolumn{6}{|c|}{ Testes (triagem) } \\
\hline & \multicolumn{2}{|c|}{ HBsAg } & \multicolumn{2}{|c|}{ Anti-HCV } & \multicolumn{2}{|c|}{ Anti-HBc } \\
\hline & $\mathrm{n}^{0}$ & $\%$ & $\mathrm{n}^{0}$ & $\%$ & $\mathrm{n}^{0}$ & $\%$ \\
\hline Positivo & 164 & 0,6 & 298 & 1,2 & 2.250 & 8,7 \\
\hline Negativo & 25.727 & 99,4 & 25.593 & 98,8 & 23.641 & 91,3 \\
\hline Total & 25.891 & 100,00 & 25.891 & 100,0 & 25.891 & 100,0 \\
\hline
\end{tabular}

Por diferentes razões, 91 (55,5\%) indivíduos entre os 164 com presença de HBsAg nos testes de triagem não tiveram concluída a investigação com vistas à confirmação. Dos 73 restantes que compareceram ao Ambulatório de Hepatites, ocorreu confirmação em 57 (Tabela 3), correspondendo a uma prevalência igual a $0,2 \%\left(\mathrm{IC}_{95 \%}: 0,16-0,28\right)$. 0 valor foi aproximadamente três vezes mais elevado entre os doadores do sexo masculino $(0,3 \%)$, em comparação ao feminino $(0,1 \%)$. A análise estatística mostra um valor borderline de probabilidade: $\chi_{(1 \mathrm{GL})}^{2}=3,6955 ; \mathrm{p}=0,055$.

Entre os 298 indivíduos com testes de triagem positivos ao anti-HCV ocorreu também uma perda significativa em termos de possibilidade de confirmação, com 175 (58,7\%) deles não tendo retornado ao HCFMRP-USP. Nos 123 restantes que compareceram ao $\mathrm{AH}$, a positividade foi confirmada em 81 (Tabela 4), correspondendo a uma prevalência de $0,3 \%$

Tabela 3 - Distribuição da positividade para o HBsAg entre os primodoadores que compareceram ao Ambulatório de Hepatites (teste confirmatório), segundo o sexo. Hemocentro de Ribeirão Preto, 1996/2001.

\begin{tabular}{|c|c|c|c|c|c|c|}
\hline \multirow[b]{3}{*}{ Sexo } & \multicolumn{6}{|c|}{ HBsAg } \\
\hline & \multicolumn{2}{|c|}{ positivo } & \multicolumn{2}{|c|}{ negativo } & \multicolumn{2}{|c|}{ total } \\
\hline & $\mathrm{n}^{0}$ & $\%$ & $\mathrm{n}^{0}$ & $\%$ & $\mathrm{n}^{0}$ & $\%$ \\
\hline Feminino & 4 & 0,1 & 4.239 & 99,9 & 4.243 & 100,0 \\
\hline Masculino & 53 & 0,3 & 21.504 & 99,8 & 21.557 & 100,0 \\
\hline Total & 57 & 0,2 & 25.743 & 99,8 & 25.800 & 100,0 \\
\hline
\end{tabular}

Tabela 4 - Distribuição da positividade para o anti-HCV entre os primodoadores que compareceram ao Ambulatório de Hepatites (testes confirmatórios), segundo o sexo. Hemocentro de Ribeirão Preto, 1996/ 2001.

\begin{tabular}{|c|c|c|c|c|c|c|}
\hline \multirow[b]{3}{*}{ Sexo } & \multicolumn{6}{|c|}{ Anti-HCV } \\
\hline & \multicolumn{2}{|c|}{ Positivo } & \multicolumn{2}{|c|}{ Negativo } & \multicolumn{2}{|c|}{ Total } \\
\hline & $\mathrm{n}^{0}$ & $\%$ & $\mathrm{n}^{0}$ & $\%$ & $\mathrm{n}^{0}$ & $\%$ \\
\hline Feminino & 11 & 0,3 & 4.228 & 99,7 & 4.239 & 100,0 \\
\hline Masculino & 70 & 0,3 & 21.407 & 99,7 & 21.477 & 100,0 \\
\hline Total & 81 & 0,3 & 25.635 & 99,7 & 25.716 & 100,0 \\
\hline
\end{tabular}

$\left(\mathrm{IC}_{95 \%}: 0,24-0,38\right)$. Os valores foram praticamente iguais $(0,3 \%)$ entre homens e mulheres, com a análise estatística não revelando diferenças entre os sexos: $\chi_{(1 \mathrm{GL})}^{2}=0,4976 ; \mathrm{p}=0,481$.

\section{DISCUSSÃo}

A triagem sorológica realizada nas unidades hemoterápicas não confirma a positividade para as hepatites $\mathrm{B}$ e $\mathrm{C}$, servindo apenas para excluir bolsas potencialmente infectantes. Assim, doadores positivos deverão ter sua positividade confirmada por técnicas de alta especificidade, o que não constitui uma obrigação legal desses serviços. Por essa razão, no âmbito do HCFMRP-USP os doadores positivos são encaminhados ao NVE e ao AH, para investigação, confirmação e acompanhamento.

O significativo predomínio de doadores do sexo masculino $(83,6 \%)$ é um achado consistente com o descrito em outras regiões do Brasil, com valores iguais a $81,5 \%$ no Norte, $82,7 \%$ no Nordeste, $76,6 \%$ no Centro-Oeste e $71,7 \%$ no Sul${ }^{1}$.

Apesar da evidência de que a imunização ativa com 0 anti$\mathrm{HBc}$ possa originar resposta imune, esse marcador não é tido como anticorpo neutralizante e sua presença não assinala a recuperação da infecção pelo $\mathrm{VHB}^{16}$. Mesmo assim, a exclusão de doadores anti-HBc positivos é assunto muito controverso e que gera discussões acaloradas, devido a evidência de resultados falso-positivos e o elevado número de doadores que são recusados. Com efeito, 2.250 indivíduos apresentaram presença deste marcador nos testes de triagem, o que correspondeu a uma positividade de $8,7 \%$. Vale mencionar que os doadores positivos apenas para esse marcador são considerados como definitivamente inaptos à doação, embora não sejam encaminhados para acompanhamento em serviços especializados. Este fato impossibilita a determinação do percentual de falso-positivos nesta população.

Comparações de valores de prevalência de marcadores encontrados em diferentes serviços e regiões devem ser feitas com cautela, uma vez que podem ser alteradas pelo uso de técnicas sorológicas diversas e pela realização ou não de testes confirmatórios. No presente estudo, as prevalências do HBsAg e do anti-HCV (0,6\% e 1,2\%, respectivamente), nos testes de triagem, foram semelhantes às encontradas entre os doadores do Serviço de Hemoterapia do Rio de Janeiro (Hemolad) no ano de 2.000, as quais atingiram valores de $0,5 \%$ e $1,2 \%$, respectivamente ${ }^{23}$. A prevalência mais elevada do anti-HCV nos testes de triagem poderia ser devido à maior sensibilidade $\mathrm{e}$ menor especificidade desse teste. Contudo, no presente estudo verificou-se predomínio da infecção pelo VHC também nos testes confirmatórios, embora com valores bem abaixo daqueles observados na triagem (0,2\% para a hepatite B e $0,3 \%$ para a hepatite $\mathrm{C}$ ), sinalizando para a presença mais comum da hepatite C na população de estudo. Tal achado foi também observado entre primodoadores da Cruz Vermelha Americana, de 1996 a 2001, com as prevalências do anti-HCV invariavelmente acima das correspondentes ao $\mathrm{HBsAg}^{9}$. 
Apesar da semelhança de alguns dos seus mecanismos de transmissão, presença simultânea do HBsAg e do antiHCV foi encontrada em apenas 2 doadores, ambos do sexo masculino e na faixa etária de 26 a 35 anos. Em ambos ocorreu presença também do anti-HBc. Ao mesmo tempo, observou-se que, dos 164 doadores positivos para o HBsAg, 136 apresentaram positividade concomitante para 0 anti-HBc.

Embora não mostrado em tabelas, nos testes de triagem ocorreram maiores valores de positividade, para ambas as hepatites virais, entre doadores do sexo masculino. Este fato poderia indicar maior frequiência de exposição a potenciais fatores de risco entre os homens, confirmando os resultados de estudos que apontam nessa direção, tal como o efetuado em duas regiões de Mato Grosso ${ }^{26}$. Todavia, a análise dos testes confirmatórios mostrou um valor de probabilidade borderline em relação à hepatite $\mathrm{B}$ e ausência de associação com o sexo em relação à hepatite $\mathrm{C}$, deixando aberta a possibilidade de que riscos diferenciados ocorram em doadores do sexo masculino apenas em relação à infecção pelo VHB. É possível que tal achado seja decorrente de riscos diferenciados ligados à transmissão por via sexual - geralmente mais comuns entre indivíduos do sexo masculino - e ao esperado baixo número de usuários de drogas ilícitas injetáveis entre doadores de sangue de ambos os sexos. Essa idéia parece ser corroborada pelo achado de associação entre o HBsAg e faixas etárias mais jovens, o que não ocorreu com os testes para a hepatite $\mathrm{C}$ (dados não mostrados em tabelas). A propósito, mesmo sem apresentar significância estatística, verificou-se que a faixa etária em que a hepatite $C$ apresentou prevalência mais elevada foi a de 46 a 60 anos, o que poderia estar associado a transfusões antes que o controle sorológico para o VHC fosse estabelecido como rotina $^{12}$.

Vale ressaltar os reduzidos valores observados no presente estudo, indicativos da baixa circulação dos vírus B e C na população de doadores de sangue do Hemocentro de Ribeirão Preto. Com efeito, as prevalências de marcadores para ambos os vírus nos testes de triagem situam-se muito abaixo das referidas, no início da década de 1990, entre doadores de Campinas $^{11}$ (1,5\% para o HBsAg, 2,6\% para 0 anti-HCV e $11 \%$ para 0 anti-HBc), embora as diferenças entre as técnicas utilizadas nos dois estudos dificultem a comparação. Cotejado com uma investigação recente realizada no México ${ }^{4}$, utilizando testes semelhantes aos usados neste estudo, os valores evidenciados na triagem em Ribeirão Preto mostram-se semelhantes para 0 anti-HCV $(1,1 \%$ e 1,2\%, respectivamente) e inferiores para o HBsAg ( $1,1 \%$ e $0,6 \%$, respectivamente). Por outro lado, as prevalências reveladas pelos testes confirmatórios em Ribeirão Preto situam-se também muito próximas dos valores descritos em testes de triagem entre doadores gregos ${ }^{13}(0,4 \%$ para o HBsAg e 0,4\% para 0 anti-HCV). Particularizando para 0 vírus C, a prevalência observada nos testes confirmatórios no presente estudo é praticamente a mesma descrita no Reino Unido ${ }^{21}(0,4 \%)$, corroborando a idéia da baixa endemicidade desta virose entre os doadores de sangue de Ribeirão Preto.

Por fim, merece um comentário o achado de elevados percentuais de não retorno para confirmação sorológica por parte de indivíduos cujos testes de triagem para hepatites virais mostraram resultados reagentes. Estas perdas atingiram $55,5 \%$ dos positivos ao HBsAg e $58,7 \%$ dos positivos ao antiHCV, sobre os quais nada se sabe em termos de serem ou não infectados. Por sua evidente relevância, essas perdas fazem por merecer uma análise mais detalhada acerca dos seus determinantes e de possíveis soluções, o que será objeto de publicação específica.

\section{REFERÊNCIAS BIBLIOGRÁFICAS}

1. Agência Nacional de Vigilância Sanitária. Relatório de produção de 2000 . Disponível em: http://www.anvisa.gov.br/sangue/hemoterapia/produção.htm. Acesso em: 4 nov 2002.

2. Alter MJ. Epidemiology of hepatitis C. Hepatology 26(Suppl 1): 62S-65S, 1997.

3. Benenson AS. Hepatites Víricas. In: Benenson AS (ed) El control de las enfermidades transmissibles en el hombre. Organización Panamericana de la Salud; Washington, DC, p.262-280, 1992.

4. Carreto-Velez MA, Carrada-Bravo T, Martinez-Magdaleno A. Seroprevalence of HBV, HCV and HIV among blood donors in Irapuato, Mexico. Salud Publica de Mexico 45 (supl):S690-S693, 2003.

5. Carvalho HB, Mesquita F, Massad E, Bueno RC, Lopes GT, Ruiz MA, Burattini MN. HIV and infections of similar transmission patterns in a drug injectors community of Santos, Brazil. Journal of Acquired Immune Deficiency Syndromes and Human Retrovirology 12: 84-92, 1996.

6. Centers for Diesease Control and Prevention. Prevención contra Hepatitis B. Disponível em: http://www.cdc.gov/spanish/enfermedades/hepatitisB.htm. Acesso em: 27 jul 2001.

7. Dean AG, Dean JA, Coulombier D, Brendel KA, Smith DC, Burton AH, Dicker RC, Sullivan K, Fagan RF, Arner TG. Epi Info, Version 6: a word processing, database, and statistics program for public health on IBM-compatible microcomputers. Centers for Disease Control and Prevention, Atlanta, 1994.

8. Diário Oficial da União. Estabelece a obrigatoriedade do cadastramento dos doadores de sangue, bem como a realização de exames laboratoriais no sangue coletado, visando a prevenir a propagação de doenças, e dá outras providências. Diário Oficial da União de 27 de janeiro de 1988. Lei Federal nº 7.649 de 25 de Janeiro de 1988, Brasília, 1988.

9. Dodd RY, Notari IV EP, Stramer SL. Current prevalence and incidence of infectious disease markers and estimated window-period risk in the American Red Cross blood donor population. Transfusion 42: 966-972, 2002.

10. Focaccia R. Prevalência das hepatites virais A, B, C e E - Estimativa da prevalência na população geral da cidade de São Paulo, medida por marcadores séricos, em amostragem populacional estratificada com sorteio aleatório e coleta domiciliar. Tese de Doutorado, Universidade de São Paulo, São Paulo, SP, 1997.

11. Gonçales Jr FL, Boccatto RS, Pedro RJ, Papaiordanou PM, Souza CA, Gonçales NS, Pellegrino Jr J. Prevalências do HBsAg, do anti-HBs e do anti-HCV na população de candidatos a doadores de sangue do Hemocentro-Campinas. Revista do Instituto de Medicina Tropical de São Paulo 35:45-51, 1993.

12. Guerrero Romero JF, Castañeda A, Rodrígues Morán M. Prevalencia y factores de riesgo associados a hepatitis $\mathrm{C}$ em donadores de sangre em el município de Durango, México. Salud pública de México 38: 94-100, 1996.

13. Koulentaki M, Spanoudakis S, Kantidaki E, Drandakis P, Tzagarakis N, Biziagos E, Moschandrea J, Kouroumalis EA. Prevalence of hepatitis B and $\mathrm{C}$ markers in volunteer blood donors in Crete. A 5-year study. Journal of Viral Hepatitis 6: 243-248, 1999.

14. Lefrere JJ. Descriptive epidemiology of hepatitis C virus infection in France in 1996. Transfusion Clinique et Biologique 4: 299-319, 1997.

15. Martinelli ALC, Barone AA, Gonçalves Jr FL, Carrilho FJ, Porta G, Sette Jr H, Silva JM, Silva LJ, Villanova MG, Ferraz MLG, Azevedo RA, Boccato RSMS, 
Lorenço R, Antunes SV. Clínica-Uniformização de política para acompanhamento e tratamento das hepatites virais B e C no Estado de São Paulo. In: Manual de Vigilância Epidemiológica. Hepatites Virais, Normas e Instruções. São Paulo: CVE - Centro de Vigilância Epidemiológica Professor Alexandre Vranjac/Secretaria de Estado da Saúde, 2000.

16. Milich DR. Immune response to hepatitis B vírus proteins: relevance of the murine model. Seminars in Livers Disease 11: 93-120, 1991.

17. Ministério da Saúde. Aprova alterações na Portaria n ${ }^{0} 721 / \mathrm{GM}$ de 09/08/ 89, que aprova Normas Técnicas para coleta, processamento e transfusões de sangue, componentes e derivados, e dá outras providências. Diário Oficial da União de 02 de dezembro de 1993 . Portaria $\mathrm{n}^{\circ} 1.376$ de 19 de novembro de 1993, 1993.

18. Ministério da Saúde. Determina que constituem objeto de notificação compulsória, em todo o território nacional, as doenças relacionadas. Diário Oficial da União de 23 de dezembro de 1999. Portaria n ${ }^{\circ} 1.461$ de 22 de dezembro de 1999, 1999.

19. Ministério da Saúde. Aprova o Regulamento Técnico para a obtenção, testagem, processamento e Controle de Qualidade de sangue para uso humano. Diário Oficial da União 19 de dezembro de 2002. Resolução RDC $n^{\circ} 343$ de 13 de dezembro de 2002, 2002

20. Ministério da Saúde. Institui no âmbito do SUS, o Programa Nacional para a Prevenção e 0 Controle das Hepatites Virais. Diário Oficial da União de 6 de fevereiro de 2002. Portaria $\mathrm{n}^{0} 263$ de 5 de fevereiro de 2002, 2002.
21. Mutimer DJ, Harrison RF, O'Donnell KB, Shaw J, Martin BA, Atrah H, Ala FA, Skidmore S, Hubscher SG, Neuberger JM. Hepatitis C infection in the assymptomatic British blood donor. Journal of Viral Hepatitis 2:47-53, 1995.

22. Ockner RK. Hepatite viral aguda. In: Wyngaarden JB, Smith Jr L, Bennett JC (eds) Cecil - Tratado de medicina interna. 19a edição, Guanabara-Koogan, Rio de Janeiro, p.780-787, 1993.

23. Santos MC, Siqueira IM, Mendes RLC. Freqüência de marcadores sorológicos positivos em doadores de sangue no Hemolad - Serviço de Hemoterapia - Janeiro a Dezembro de 2.000. Série de Monografias da Escola Brasileira de Hemoterapia 8(supl): 189, 2001.

24. Sherlock S, Dooley J. Diseases of the liver and biliary system. $10^{\text {th }}$ edição London: Blackwell Science 274-294, 1997.

25. Silva AO. Tratamento da hepatite $\mathrm{C}$ e de suas formas evolutivas - Breviário Pizarro Farmacêutica, São Paulo, 2000.

26. Souto FJD. Estudo Epidemiológico sobre a infecção pelo vírus da hepatite B em duas regiões do Estado de Mato Grosso. Tese de Doutorado. Faculdade de Medicina. Universidade Federal de Minas Gerais. Belo Horizonte, MG, 1997

27. StataCorp. Stata Statistical Software: Release 6.0 College Station, Stata Corporation, Texas, 1999.

28. Torres JR. Hepatitis B and hepatitis delta virus infection in South America. Gut 38(suppl): 48S-55S, 1996. 